\title{
Equimomental Tetrads of a Rigid Body.
}

By D. M. Y. Sommerville.

A lamina of mass $m$ can be replaced by an equimomental system of three equal particles $\frac{1}{3} m$ placed at the vertices of a maximum inscribed triangle of a momental ellipse for the centre of mass. Similarly any rigid body can be replaced by an equimomental system of four equal particles (cf. Routh, Elementary Rigid Dynamics, 7th ed. (1905), Art. 44 and Note, p. 423).

The following proof is based on the relations between two sets of four rectangular axes in space of four dimensions.

Take as coordinate-axes the principal axes at the centre of mass, and let the moments of inertia w. r. t. the coordinate-planes be $A, B, C$. Let $\left(x_{i}, y_{i}, z_{i}\right) \quad(i=1,2,3,4)$ be the coordinates of four particles. each of mass $\frac{1}{4} m$. We have then

and

$$
\begin{aligned}
& \frac{1}{4} m \sum x_{i}^{2}=A, \text { etc. } \\
& \sum y_{i} z_{i}=0, \text { etc. }
\end{aligned}
$$

Also since the centre of mass is at the origin, $\Sigma x_{i}=0$, etc.

$$
\text { Write } \frac{1}{4} m x_{i}^{2} / A=l_{i}^{2}, \quad \frac{1}{4} m y_{i}^{2} / B=m_{i}^{2}, \quad \frac{1}{4} m z_{i}^{2} / C=n_{i}^{2},
$$

and

$$
\frac{1}{4}=p_{i}^{2} \quad(i=1,2,3,4) .
$$

Then we have $\Sigma l_{i}^{2}=1, \quad \Sigma m_{i}^{2}=1, \quad \Sigma n_{i}^{2}=1, \quad \Sigma p_{i}^{2}=1$, $\Sigma l_{i} m_{i}=0, \Sigma l_{i} n_{i}=0, \sum l_{i} p_{i}=0$, and three other similar equations.

Hence $\left(l_{1}, l_{2}, l_{3}, l_{4}\right)$, etc., are direction-cosines of four mutually rectangular axes $O, X^{\prime} Y^{\prime} Z^{\prime} W^{\prime}$ in space of four dimensions referred to rectangular axes $O, X Y Z W$. The direction-cosines of $O X$ referred to $O, X^{\prime} Y^{\prime} Z^{\prime} W^{\prime}$ are then $\left(l_{1}, m_{1}, n_{1}, p_{1}\right)$, and similarly for the others. Hence we have

$$
\begin{array}{cc} 
& l_{i}^{2}+m_{i}^{2}+n_{i}+p_{i}^{2}=1 \quad(i=1,2,3,4) ; \\
\text { i.e. } \quad & \frac{1}{4} m\left(x_{i}^{2} / A+y_{i}^{2} / B+z_{i}^{2} / C\right)+\frac{1}{4}=1 \\
\text { or } & x_{i}^{2} / A+y_{i}^{2} / B+z_{i}^{2} / C=3 / m .
\end{array}
$$

Also

$$
l_{i} l_{j}+m_{i} m_{j}+n_{i} n_{j}+p_{i} p_{j}=0 \quad(i \neq j=1,2,3,4) ;
$$

hence $\quad x_{i} x_{j} / A+y_{i} y_{j} / B+z_{i} z_{j} / C=-1 / m$. 
The four points therefore form a tetrahedron inscribed in the ellipsoid

$$
x^{2} / A+y^{2} / B+z^{2} / C=3 / m,
$$

and self-conjugate w.r.t. the homothetic and concentric virtual ellipsoid

$$
x^{2} / A+y^{2} / B+z^{2} / C=-1 / m ;
$$

whence we deduce that they form a circumscribed tetrahedron of the real ellipsoid

$$
\frac{x^{2}}{A}+\frac{y^{2}}{B}+\frac{z^{2}}{C}=\frac{1}{3 m}
$$

These ellipsoids are similar to Legendre's equimomental ellipsoid.

\title{
The Number of Lines that may lie upon a Surface of given Order.
}

\author{
By H. W. Richmond.
}

The greatest number of straight lines that can lie upon a surface of order $n$ (not being a ruled surface) is unknown, except if $n$ is three. Salmon and Clebsch have shown that the points of contact of lines which have a four-point contact with the surface lie upon a locus of order $n(11 n-24)$, the intersection of the surface of order $n$ with another of order $11 n-24$. Since a straight line lying wholly on the former surface must form a part of this locus, the number $n(11 n-24)$ is an upper limit to the number of lines; if $n$ is three, this gives 27, the correct number. But for values of $n>3$, it is improbable that this limit ${ }^{1}$ can be reached.

At the Colloquium, held at St Andrews in July 1930, the surfaces

$$
\text { (i) } x^{3}+y^{3}+z^{3}+t^{3}=0 \text {; (ii) } x^{4}+y^{4}=u^{4}+v^{4} \text {; }
$$

were considered, and a question was asked as to the number of lines lying on each. By a generalization of the result a theorem is obtained in regard to surfaces of any order on which lie an unexpectedly large number of lines.

"There exist surfaces of order $n$, not ruled and without conical points, on which lie $3 n^{2}$ straight lines; of these $n(n+2)$ may be real."

${ }^{1}$ See Encyklopïdie d. math. Wissenschaften, Band III, Teil 2, p. 665. 